Tropical Journal of Pharmaceutical Research April 2021; 20 (4): 727-733

ISSN: $1596-5996$ (print); 1596-9827 (electronic)

(C) Pharmacotherapy Group, Faculty of Pharmacy, University of Benin, Benin City, 300001 Nigeria.

Available online at http://www.tjpr.org

Original Research Article

http://dx.doi.org/10.4314/tjpr.v20i4.10

\title{
Up-regulation of miR-20a ameliorates sevoflurane anesthesia-induced cognitive impairment in rats by targeting EphA4
}

\author{
Duo Qian', Changlin Chen', Biqian Dong1, Yu Qiü,3* \\ ${ }^{1}$ Department of Anesthesiology, Affiliated Hospital of North Sichuan Medical College, Nanchong City, Sichuan Province \\ 637000, ${ }^{2}$ Department of Anesthesiology, The First Hospital of Qiqihar, Qiqihar City, Heilongjiang Province 161005, ${ }^{3}$ Department \\ of Anesthesiology, Affiliated Qiqihar Hospital Southern Medical University, Qiqihar City, Heilongjiang Province 161005, China
}

*For correspondence: Email: yu_q12@163.com; Tel: +86-0452-2549847

Sent for review: 6 January 2021

Revised accepted: 7 April 2021

\begin{abstract}
Purpose: To evaluate the role of miR-20a in sevoflurane (SEV)-induced cognitive impairment in rats and to elucidate the mechanism of action.

Methods: A SEV-induced cognitive impairment rat model was developed. The Morris water maze test and fear assay were carried out to assess impaired learning and memory. A cellular SEV-impaired model was developed and the miR-20a level was measured in the animal and cellular models. TUNEL staining and immunoblot assay were performed to determine the SEV effect on apoptosis. Bioinformatic analysis and luciferase assay were conducted to identify the target of miR-20a action. A rescue assay involving miR-20a overexpression in cellular and animal models was developed and used to evaluate function of miR-20a in cognitive defects.

Results: The rats showed significant cognitive impairment upon SEV treatment, which inhibited the expression of miR-20a and promoted neuronal apoptosis. Further findings identified EphA4 as a target of miR-20a, which regulates its expression. Overexpression of miR-20a in rats effectively reduced cognitive dysfunction and apoptosis of hippocampus somatic cells caused by SEV treatment.

Conclusion: Evidently, miR-20a ameliorates SEV anesthesia-induced cognitive impairment in rats and thus has the potential to serve as a therapeutic target for the treatment of post-operative cognitive dysfunction.
\end{abstract}

Keywords: Postoperative cognitive dysfunction (POCD), miR-20a, Sevoflurane, EphA4, Apoptosis

\begin{abstract}
This is an Open Access article that uses a fund-ing model which does not charge readers or their institutions for access and distributed under the terms of the Creative Commons Attribution License (http://creativecommons.org/licenses/by/4.0) and the Budapest Open Access Initiative (http://www.budapestopenaccessinitiative.org/read), which permit unrestricted use, distribution, and reproduction in any medium, provided the original work is properly credited.
\end{abstract}

Tropical Journal of Pharmaceutical Research is indexed by Science Citation Index (SciSearch), Scopus, International Pharmaceutical Abstract, Chemical Abstracts, Embase, Index Copernicus, EBSCO, African Index Medicus, JournalSeek, Journal Citation Reports/Science Edition, Directory of Open Access Journals (DOAJ), African Journal Online, Bioline International, Open-J-Gate and Pharmacy Abstracts

\section{INTRODUCTION}

Postoperative cognitive dysfunction (POCD) is a common complication after major surgery that is mainly characterized by mental disorder, anxiety, personality change, and impaired memory [1]. These symptoms can last for days or weeks, but in severe cases, they can last for several months [2]. The occurrence of POCD is closely related to the anesthesia used during the operation [3]. POCD was first proposed by Bedford in 1955 and was defined as "the brain damage caused by anesthesia in the elderly" [4]. The precise pathogenesis of POCD is still unknown today. 
Sevoflurane (SEV) inhalation anesthesia has been used widely in recent years [5]. Increased attention has been paid to studying the effects of SEV inhalation on learning and memory impairment [6]. In recent years, it has been shown that SEV causes obvious memory damage in animals [7]. Although SEV has a significant effect on embryonic brain development, the mechanism is still unclear. Studies have shown that exposure to SEV during pregnancy can lead to impaired learning and memory in offspring and that this effect may be related to the concentration and duration of inhalation of SEV [8].

MicroRNAs (miRNAs) are a class of small noncoding RNAs that can bind to the 3'-untranslated region (3'-UTR) of the transcript of their homologous target genes, thereby regulating their expression [9]. Extensive studies have confirmed a broad association between miRNAs and anesthesia-related cognitive impairment [911]; miRNAs, such as miR-665, miR-572, and miR-181, have been reported to affect POCD progression, suggesting that these miRNAs could serve as promising therapeutic targets for treating POCD [12].

\section{EXPERIMENTAL}

\section{SEV-treated rat model}

All animal experiments in this study were approved by the Ethics Committee of Affiliated Hospital of North Sichuan Medical College (Approval no.2019096) and conducted in accordance with the National Institutes of Health Laboratory Animal Care and Use Guidelines [13]. Male Sprague Dawley rats $(250 \pm 10 \mathrm{~g}, 7$ weeks old, purchased from Vital River) were housed with free access to water and food. Then the rats were randomly divided into four groups: the sham group with normal air for $6 \mathrm{~h}(\mathrm{n}=10)$, the SEV group with $2.5 \%$ SEV at $600 \mu \mathrm{g} / \mathrm{kg} / \mathrm{min}$ in $100 \% \mathrm{O}_{2}$ for $6 \mathrm{~h}(\mathrm{n}=10)$, the negative control (NC) agomir group with caudal vein injection of 1 nmol miR-20a NC packed in liposomes, and the miR-20a agomir group with caudal vein injection of $1 \mathrm{nmol}$ miR-20a agomir (synthesized by GenePharma, China). The miRNA agomir was packed into liposomes and injected every 3 weeks for 15 weeks. Then the rats were allowed to recover for 7 days before further experimentation.

\section{Morris water maze test}

To perform the Morris water maze (MWM) test, rats were forced to swim and locate a hidden platform in a rectangular channel. Rats were initially placed on the platform to become familiar with its location in the channel. One day later, the rectangular maze was changed into a circular water maze that was $1.0 \mathrm{~cm}$ beneath the surface of the water. During the experiment, rats were placed in different locations in the water. All rats were allowed to remain in the water for $70 \mathrm{~s}$ at most. If the rats failed, they were allowed to adjust to the surroundings by being placed in the quadrant opposite the target quadrant. Thereafter, the rats were trained to find a visible platform. Rats were trained four times each day. Each trial had a different platform and starting location. The escape latency times for each rat was recorded.

\section{Fear conditioning}

Each animal was placed in a chamber that smelled of $70 \%$ alcohol and subjected to three tone-foot shock pairings (tone: $2000 \mathrm{~Hz}, 85 \mathrm{db}$, $30 \mathrm{~s}$; foot shock: $1 \mathrm{~mA}, 2 \mathrm{~s}$ ) with an inter-trial time of $1 \mathrm{~min}$ in a slightly darkened room. At $30 \mathrm{~s}$ after the conditioning training, the animal was removed from this test chamber. Then $24 \mathrm{~h}$ later, the rats were returned to the chamber for $8 \mathrm{~min}$. The amount of time the animal exhibited freezing behavior was recorded in $8 \mathrm{~s}$ intervals. Then the animal was placed in a second chamber that was wiped with $1 \%$ acetic acid to establish a different context and smell from the first test chamber. Freezing behavior was noted for $3 \mathrm{~min}$. The auditory stimulus was applied for three cycles, each of which consisted of $30 \mathrm{sec}$ of stimulus followed by a $1 \mathrm{~min}$ interval. Freezing behavior was recorded for $5 \mathrm{~min}$.

\section{Quantitative PCR assay}

This experiment was performed as described previously [14]. Briefly, total RNA was extracted from rat hippocampus neuronal cells with the miRNeasy Mini Kit (Qiagen, Dusseldorf, Germany). Then, RNA was reverse-transcribed into cDNA using M-MLV reverse transcriptase with specific reverse transcription (RT) primers. Then, SYBR Green PCR Master Mix (Applied Biosystems, Thermo Fisher Scientific, USA) was used to measure the expression levels of miR20a. U6 was used as an internal control for miR20a. The RT primers used are listed in Table 1.

\section{Cell culture and cell transfection}

The HT22 mouse hippocampal neuronal cell line (ATCC, Rockville, MD, USA) was incubated in Dulbecco's Modified Eagle's Medium (DMEM) supplemented with $15 \%$ fetal bovine serum (Invitrogen, Shanghai, China). 
Table 1: Sequences of RT primers used in quantitative PCR assay

\begin{tabular}{lll}
\hline Gene & Forward primers (5'-3') & Reverse primers (5'-3') \\
\hline miR-20a & GCCCGCTAAAGTGCTTATAGTG & CCAGTGCAGGGTCCGAGGT \\
U6 & TGCGGGTGCTCGCTTCGGCAGC & CCAGTGCAGGGTCCGAGGT \\
\hline
\end{tabular}

HT22 cell exposure to SEV was performed in a gas-tight chamber at $37^{\circ} \mathrm{C}$, and $1 \% 2 \mathrm{~T}$ and $5 \%$ SEV were administered respectively by a SEVspecific vaporizer (Yu Yan, China).

The miR-20a inhibitor (miR-20a inhibitor) and its negative control (miR inhibitor-NC), as well as the miR-20a mimic (miR mimic) and its control (miR mimic-NC), were synthesized by GenePharma (Shanghai, China). HT22 cells were transfected with the oligonucleotides above using Lipofectamine 2000 reagent (Thermo Fisher Scientific).

\section{TUNEL staining}

Cells were fixed with $4 \%$ paraformaldehyde. After washing three times with PBS, the cells were stained with the Cell Death Detection Kit (SigmaAldrich, St. Louis, MO, USA). Nuclei were stained with DAPI. The TUNEL-stained images were captured using a Nikon Labophot 2 microscope.

\section{Immunoblot analysis}

Cell lysate was harvested using lysis buffer containing $50 \mathrm{mM}$ Tris ( $\mathrm{pH} 7.4$ ), $150 \mathrm{mM} \mathrm{NaCl}$, $1 \%$ NP-40, $0.5 \%$ sodium deoxycholate, and $0.1 \%$ SDS. Protein samples were resolved by SDSPAGE and transferred onto polyvinylidene fluoride membranes. Then the membranes were blocked with $5 \%$ BSA for $1 \mathrm{~h}$. Subsequently, primary antibodies against $\operatorname{Bax}(1: 1000,2774$; Cell Signaling Technology [CST], Danvers, MA, USA), Bcl-2 (1:1000, 3498; CST), cleavedcaspase-3 (1:1000, 9661; CST), EphA4 (1:1000, 8793; CST), and $\beta$-actin $(1: 1000,4970 ;$ CST) were incubated with the membrane overnight at $4^{\circ} \mathrm{C}$. Then the membranes were incubated with secondary antibody conjugated with horseradish peroxidase $(1: 1000,7074 ;$ CST). Protein bands were detected using a chemical luminescence reagent (Pierce, Rockford, IL, USA).

\section{Luciferase reporter assay}

The 3'-UTR region of the EphA4 gene was amplified using PCR and cloned into the pMIRREPORT Luciferase vector (Ambion, Thermo Fisher Scientific). The EphA4-MUT reporter was constructed using a Site-Directed Mutagenesis Kit. Then, HEK293 cells were transfected with the pMIR-REPORT vector, EphA4-WT or EphA4-
MUT reporter, and the miR-20a mimic, mimic$\mathrm{NC}, \mathrm{miR}$ inhibitor-NC or miR-20a inhibitor. After transfection, luciferase activity was measured.

\section{Statistical analysis}

Data were analyzed using SPSS 13.0 (SPSS Inc., Chicago, Illinois, USA). All data are presented as the mean \pm standard deviation (SD) $(n=3)$. The Student's t-test and analysis of variance (ANOVA) were performed to assess statistical significance, which was defined as $p<$ 0.05 .

\section{RESULTS}

\section{SEV-induced memory impairment inhibits miR-20a level in rat hippocampus}

To determine the memory and spatial learning ability of rats following SEV administration, the Barnes maze test and fear conditioning assay were conducted. As shown in Figure $1 \mathrm{~A}$, rats in the SEV group exhibited impaired cognitive ability. Simultaneously, SEV-exposed rats displayed reduced freezing behavior when compared with control rats in the contextual fear conditioning test. Freezing behavior between the SEV-treated and control rats differed minimally during the tone fear conditioning test (Figure 1 B). The miRNA-20 level was also monitored in the two groups. Interestingly, we noticed a reduced miR-20a level in SEV-treated rats (Figure $1 \mathrm{C}$ ), suggesting that SEV treatment might suppress miR-20a level in the hippocampus of rats.
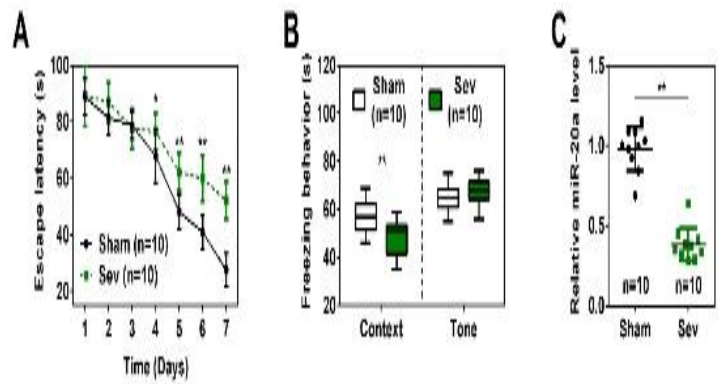

Figure 1: SEV-induced memory impairment inhibits miR-20a level in hippocampus of rats. (A) The escape latency in sham or SEV-induced rats. (B) The freezing behavior in sham or SEV-induced rats. ${ }^{*} P<0.05,{ }^{* *} p$ $<0.01$ compared with the control group

Trop J Pharm Res, April 2021; 20(4): 729 
SEV promotes neuronal apoptosis and inhibits miR-20a in vitro

HT22 cells exposed to varying concentrations of SEV were subjected to TUNEL staining to assess apoptosis. SEV increased the TUNELpositive cell number in a dose-dependent manner (Figure $2 \mathrm{~A}$ ). Meanwhile, SEV treatment upregulated the protein level of Bax-2 and cleaved Caspase- 3 and inhibited $\mathrm{Bcl}-2$ in a dosedependent manner (Figure 2 B). Similarly, miR20a was decreased by SEV exposure (Figure 2 C). These data suggest that SEV induces neuronal apoptosis in vitro.

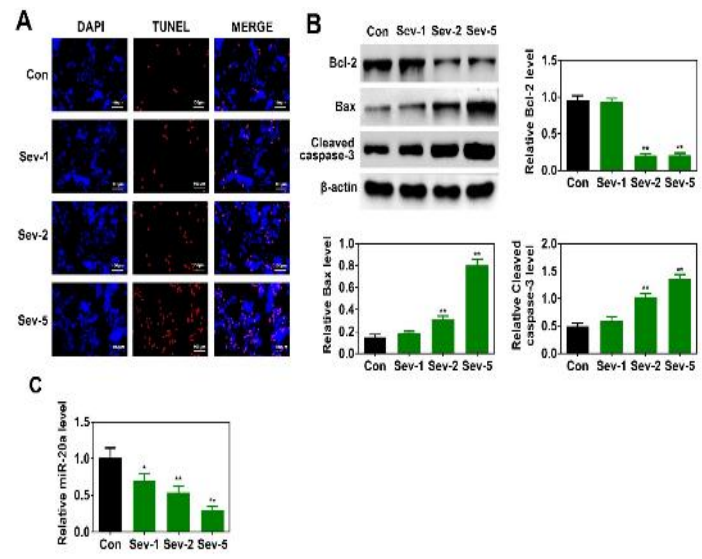

Figure 2: SEV promotes neuronal apoptosis and inhibits miR-20a in vitro. (A) TUNEL staining of HT22 cells increased with SEV concentration. (B) The level of apoptosis-related proteins was examined in SEVtreated cells by immunoblot. (C) qPCR assay measured the relative miR-20a level in HT22 cells. ${ }^{*} P$ $<0.05,{ }^{* *} p<0.01$ compared with the control group

\section{MiR-20a overexpression suppresses SEV- induced neuronal apoptosis}

To unveil the function of miR-20a in SEV-induced cell apoptosis, we examined the effect of miR20a overexpression on HT22 cells. First, the efficacy of transfection of the miR-20a mimic was confirmed by qPCR. Results revealed that miR20a levels increased after transfection (Figure 3 A). Then apoptosis was examined in cells exposed to $5 \%$ SEV. The data demonstrate that increased apoptosis in SEV-treated cells was suppressed by miR-20a transfection, suggesting the protective effect of miR-20a in neurons (Figure $3 \mathrm{~B}$ ). Moreover, we assessed the level of apoptosis-related proteins in the different groups. The elevated expression level of Bax and cleaved Caspase-3 induced by SEV were inhibited by miR-20a transfection (Figure $3 \mathrm{C}$ ), further confirming that miR-20a suppressed SEVinduced apoptosis.
A

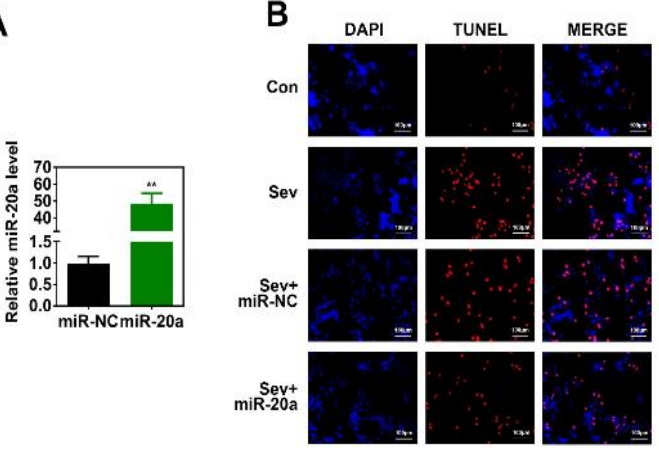

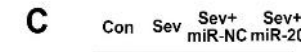
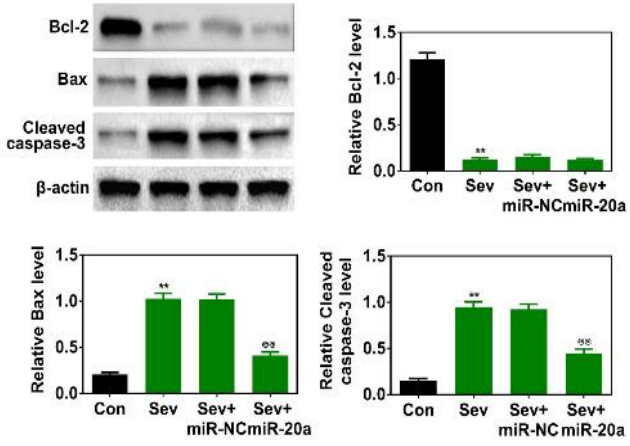

Figure 3: MiR-20a overexpression suppresses SEVinduced neuronal apoptosis. (A) Relative miR-20a levels in control or miR-20a-transfected cells were measured by qPCR. (B) TUNEL assay of SEV-treated HT22 cells in the presence of miR-20a or miR-NC overexpression. (C) Apoptosis-related protein level was assessed in SEV-treated or -untreated cells overexpressing miR-20a or miR-NC

\section{MiR-20a targets EphA4 to suppress its expression}

We screened the Targetscan database (http://www.targetscan.org/) to identify the potential target of miR-20a. A 3'-UTR of EphA4 was found to be complementary to the miR-20a sequence (Figure $4 \mathrm{~A}$ ). To verify the regulatory relationship, luciferase assays were performed. The EphA luciferase activity was inhibited or elevated in miR-20a mimic- or miR-20a inhibitortransfected cells, respectively, indicating that miR-20a targets EphA4 (Figure 4 B). EphA4 mutant luciferase activity remained unchanged in the miR-20a mimic- or miR-20a inhibitortransfected groups (Figure 4B). The level of EphA4 protein was elevated or inhibited by miR20a inhibitor or miR-20a mimic, respectively (Figure $4 \mathrm{C}$ ). Moreover, EphA4 protein level was induced in SEV-treated rat hippocampus (Figure 4D). Taken together, these data suggest that miR-20a regulated EphA4 expression. 
A

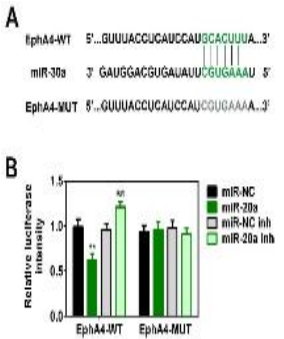

D

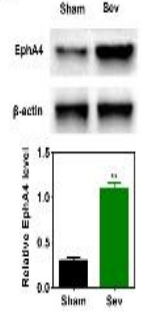

Figure 4: MiR-20a targets EphA4 to suppress its expression. (A) The predicted binding nucleotides of miR-20a and EphA4, along with the mutant sequence of predicted binding nucleotides in EphA4. (B) Luciferase assay was performed to measure the EphA4 (WT) or EphA4 (MUT) reporter activity following transfection of the indicated miR-20a mimics or miR-20a inhibitor. (C) EphA4 protein level in cells transfected with the indicated miR-20a mimics or miR20 a inhibitor. (D) EphA4 protein level in sham or SEVtreated rat hippocampus; ${ }^{* *} p<0.01$ compared with the miR-NC group; @@p $<0.01$ compared with the miR-NC inhibitor group

\section{MiR-20a overexpression reverses SEV- induced cognitive impairment}

Since miR-20a overexpression could reverse apoptosis induced by SEV, we investigated whether the cognitive defects induced by SEV could be improved by miR-20a. SEV-treated rats were subjected to miR-20a agomir or NC agomir transfection followed by the Barnes maze test and fear conditioning assay. miR-20a agomir injection reversed memory impairment and learning ability induced by SEV (Figure $5 \mathrm{~A}$ ). The decreased freezing behavior in SEV-exposed rats was also reversed by miR-20a injection in the contextual fear conditioning test. However, the freezing behavior changed only minimally among the different groups during the tone fear conditioning test (Figure $5 \mathrm{~B}$ ). In addition, EphA4 was induced by SEV exposure and was decreased by miR-20a (Figure $5 \mathrm{C}$ ).
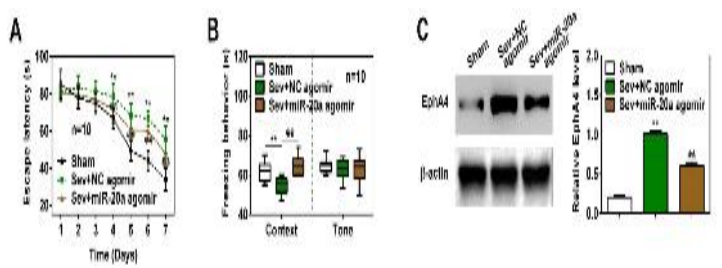

Figure 5: MiR-20a overexpression alleviates SEVinduced cognitive impairment. (A) Escape latency values in sham, SEV-induced, or miR-20a agomirinjected SEV-treated rats. (B) Freezing behavior in sham, SEV-induced, or miR-20a agomir-injected SEVtreated rats. (C) EphA4 protein levels in sham, SEVinduced, or miR-20a agomir-injected SEV-treated rats. ${ }^{* \star} P<0.01$ versus sham group; @@ $p<0.01$ compared with the SEV-treated group

\section{DISCUSSION}

POCD is a common condition that is more likely to occur in the elderly, with a morbidity of approximately $10-60 \%$ [15]. Anesthetic agents affect the central nervous system, and preoperative agents have been associated with postoperative psychiatric disorders [16]. Even though some studies have shown that, after SEV sevoflurane administration, patients have a lower degree of cognitive decline and a faster recovery time, understanding how SEV causes cognitive impairment is still worth further study [17]. In this study, a SEV anesthesia rat model of POCD was developed to simulate cognitive impairment, and our results demonstrate that miR-20a upregulation ameliorates SEV-induced cognitive impairment. These experimental results suggest a potential treatment for POCD.

Using Morris water maze and conditional fear experiments, we found that SEV-treated rats exhibit significant cognitive impairment and miR20a downregulation. SEV also promoted neuronal apoptosis as evidence by the TUNEL assay. These experiments confirm the effects of SEV on neurons and cognition, as well as demonstrate a correlation between SEV and miR-20a expression.

miR-20a is a critical miRNA involved in multiple cellular functions [18]. miR-20a promotes kidney injury in sepsis rats through autophagy, and regulates fibroblast-like synoviocyte proliferation and apoptosis in rheumatoid arthritis [19]. After SEV treatment, the expression of miR-20a was downregulated in the hippocampus of newborn rats and lungs of adult rats [20]. Importantly, miR-20a has been reported to affect cognitive function [20]. After nerve injury in rats, miR-20a levels were downregulated. Lastly, overexpression of miR-20a reversed sensory function. Therefore, it can be inferred that miR20a may play a role in SEV-induced cognitive impairment [21]. In this study, miR-20a expression was found to be decreased upon SEV treatment, and overexpression of miR-20a in rats effectively reduced the cognitive dysfunction and apoptosis of hippocampus somatic cells caused by SEV treatment. These findings further confirm the key role of miR-20a in cognitive impairment regulation.

EphA4 plays a very important role in regulating neuronal function [22]. EphA4 has been shown to be required for neural circuits controlling skilled reaching, and inhibition of the EphA4 pathway blocked trigeminal neuropathic pain [23]. EphA4 interacts with vascular endothelial growth factor receptor 2 in the neural stem and affects 
progenitor cell differentiation [24]. A previous study demonstrated that EphA4 expression was upregulated in the brain with nerve injury, and that depletion of EphA4 could improve the injury [25]. Here, we found that EphA4 is regulated by miR-20a, and therefore may regulate the progression of POCD. These data provide evidence that EphA4 may serve as a target for POCD treatment.

\section{CONCLUSION}

Rats showed cognitive impairment upon SEV treatment, which also decreased miR-20a expression and promoted neuronal apoptosis. miR-20a overexpression inhibited the apoptosis of neurons. miR-20a targets EphA4 and regulates its expression. Overexpression of $\mathrm{miR}$ $20 \mathrm{a}$ in rats reduces the cognitive dysfunction and apoptosis of hippocampus somatic cells caused by SEV treatment. Thus, these findings suggest that miR-20a is a potential therapeutic target for POCD treatment.

\section{DECLARATIONS}

\section{Conflict of interest}

No conflict of interest is associated with this work.

\section{Contribution of authors}

We declare that this work was performed by the authors named in this article and all liabilities pertaining to claims relating to the content of this article will be borne by the authors. Duo Qian designed the study and supervised the data collection. Changlin Chen and Biqian Dong analyzed and interpreted the data. Yu Qu prepared the manuscript for publication and reviewed the draft of the manuscript. All authors have read and approved the manuscript.

\section{Open Access}

This is an Open Access article that uses a funding model which does not charge readers or their institutions for access and distributed under the terms of the Creative Commons Attribution License (http://creativecommons.org/licenses/by/ 4.0) and the Budapest Open Access Initiative (http://www.budapestopenaccessinitiative.org/rea d), which permit unrestricted use, distribution, and reproduction in any medium, provided the original work is properly credited.

\section{REFERENCES}

1. Yousuf MS, Samad K, Ullah H. Postoperative Cognitive Dysfunction following General Anaesthesia in Patients Undergoing Elective Non-cardiac Surgery. J Coll Physicians Surg Pak 2020; 30(4): 417-419.

2. Zhou $X Y$, Liu J, Xu ZP, Fu Q, Wang PQ, Wang JH, Zhang $H$. Dexmedetomidine ameliorates postoperative cognitive dysfunction by inhibiting Toll-like receptor 4 signaling in aged mice. Kaohsiung J Med Sci 2020; 36(9): 721-731.

3. Chen L, Zhang S, Tian $W$, Huang L, Jiang $X$, Chen $X$, Wang $H$, Su D. Cerebral spinal fluid cholinergic biomarkers predict postoperative cognitive dysfunction in aged patients - A prospective, observational, single center study. J Clin Anesth 2020; 62(109743.

4. Gong M, Wang G, Li G, LiU J, Sun P, Xu L, Li J, Guo Y, Zhang $M$. Dysfunction of inflammation-resolving pathways is associated with postoperative cognitive decline in elderly mice. Behav Brain Res 2020; 386: 112538.

5. Bai Y, Li CS, Lu XH, Zhou Y, Miao CH. [Effects of desflurane and sevoflurane anesthesia on postoperative recovery after long-term tumor surgery]. Zhonghua $Y i$ Xue Za Zhi 2020; 100(29): 2278-2282.

6. Jung S, Na S, Kim HB, Joo HJ, Kim J. Inhalation sedation for postoperative patients in the intensive care unit: initial sevoflurane concentration and comparison of opioid use with propofol sedation. Acute Crit Care 2020; 35(3): 197-204.

7. Lin Y, Lei L, Ju LS, Xu N, Morey TE, Gravenstein N, Yang J, Martynyuk AE. Neonatal exposure to sevoflurane expands the window of vulnerability to adverse effects of subsequent exposure to sevoflurane and alters hippocampal morphology via decitabinesensitive mechanisms. Neurosci Lett 2020; 735 : 135240.

8. Zhang $N$, Jin $Y, H u$ Q, Cheng S, Wang C, Yang Z, Wang $Y$. Circular RNA hsa_circ_0078607 suppresses ovarian cancer progression by regulating miR-518a-5p/Fas signaling pathway. J Ovarian Res 2020; 13(1): 1-10.

9. Liu Q, Hou A, Zhang Y, Guo Y, Li J, Yao Y, Niu K, Li H, Ma Y, Cao J. MiR-190a potentially ameliorates postoperative cognitive dysfunction by regulating Tiam1. BMC Genomics 2019; 20(1): 670.

10. Gao R, Chen C, Zhao Q, Li M, Wang Q, Zhou L, Chen E, Chen $H$, Zhang $Y$, Cai $X$ et al. Identification of the Potential Key Circular RNAs in Elderly Patients With Postoperative Cognitive Dysfunction. Front Aging Neurosci 2020; 12(165.

11. Zhang L, Xue Z, Yan J, Jiang H. LncRNA Rik-203 Contributes to Sevoflurane Induced Neurotoxicity? Front Med (Lausanne) 2020; 7: 353.

12. Tan DX, Chen XX, Bai TZ, Zhang J, Li ZF. Sevoflurane up-regulates microRNA-204 to ameliorate myocardial ischemia/reperfusion injury in mice by suppressing Cotl1. Life Sci 2020; 259(118162. 
13. Care IOLARCo, Animals UoL: Guide for the care and use of laboratory animals: US Department of Health and Human Services, Public Health Service, National ...; 1986.

14. Chen C, Li X, Li J, Xu Y, Jing X, Wu S, LiU X, Zhang XY. Purification and characterization of an antimicrobial protein from Gastrodia elata Blume tubers. Tropical Journal of Pharmaceutical Research 2018; 17: 17171723.

15. Huang JM, LV ZT, Zhang $B$, Jiang $W X$, Nie $M B$. Intravenous parecoxib for early postoperative cognitive dysfunction in elderly patients: evidence from a metaanalysis. Expert Rev Clin Pharmacol 2020; 13(4): 451460.

16. Knaak C, Brockhaus WR, Spies C, Borchers F, Piper SK, Radtke FM, Lachmann G. Presurgical cognitive impairment is associated with postoperative delirium and postoperative cognitive dysfunction. Minerva Anestesiol 2020; 86(4): 394-403.

17. Muller J, Nowak $S$, Vogelgesang A, von Sarnowski B, Rathmann E, Schmidt S, Rehberg S, Usichenko T, Kertscho H, Hahnenkamp $K$ et al. Evaluating Mechanisms of Postoperative Delirium and Cognitive Dysfunction Following Elective Spine Surgery in Elderly Patients (CONFESS): Protocol for a Prospective Observational Trial. JMIR Res Protoc 2020; 9(2): e15488.

18. Yang H, Li Y, Peng Z, Wang Y. Overexpression of miR20 a promotes the progression of osteosarcoma by directly targeting QKI2. Oncol Lett 2019; 18(1): 87-94.

19. Wang IK, Sun KT, Tsai TH, Chen CW, Chang SS, Yu $T M$, Yen TH, Lin FY, Huang CC, Li CY. MiR-20a-5p mediates hypoxia-induced autophagy by targeting ATG16L1 in ischemic kidney injury. Life Sci 2015; 136: 133-141.

20. Feng $Y$, Duan $C$, Luo Z, Xiao W, Tian F. Silencing miR20a-5p inhibits axonal growth and neuronal branching and prevents epileptogenesis through RGMa-RhoAmediated synaptic plasticity. J Cell Mol Med 2020.

21. Chai H, Liu M, Tian R, Li X, Tang H. miR-20a targets BNIP2 and contributes chemotherapeutic resistance in colorectal adenocarcinoma SW480 and SW620 cell lines. Acta Biochim Biophys Sin (Shanghai) 2011; 43(3): 217-225.

22. Zhao J, Taylor CJ, Newcombe EA, Spanevello MD, O'Keeffe I, Cooper LT, Jhaveri DJ, Boyd AW, Bartlett PF. EphA4 Regulates Hippocampal Neural Precursor Proliferation in the Adult Mouse Brain by d-Serine Modulation of N-Methyl-d-Aspartate Receptor Signaling. Cereb Cortex 2019; 29(10): 4381-4397.

23. Cruz-Orengo L, Figueroa JD, Velazquez I, Torrado A, Ortiz C, Hernandez C, Puig A, Segarra AC, Whittemore SR, Miranda JD. Blocking EphA4 upregulation after spinal cord injury results in enhanced chronic pain. Exp Neurol 2006; 202(2): 421-433.

24. Chen Q, Liu J, Sawada T, Wei C, Wu S, Han F. Possible role of EphA4 and VEGFR2 interactions in neural stem and progenitor cell differentiation. Exp Ther Med 2020; 19(3): 1789-1796.

25. Herrmann JE, Shah RR, Chan AF, Zheng B. EphA4 deficient mice maintain astroglial-fibrotic scar formation after spinal cord injury. Exp Neurol 2010; 223(2): 582598. 